observed by Dr. Halm, at Blackford Hill, as follows: November 18 , 18h. $26 \mathrm{~m}$. 145. G.M.T.; R.A. $13 \mathrm{~h} .48 \mathrm{~m}$. I5s., Decl. $0^{\circ} 48^{\prime} 18^{\prime \prime} \cdot 8 \mathrm{~N}$. The movement of the comet is in a direction sunth-east by east, and it now rises about $4 \mathrm{a} . \mathrm{m}$. Dr. Lamp states that the comet is moderately bright, but not visible to the naked eye ; it is round, with a central condensation, and a straight tail (Ast. Nach. 3318).

Variable Star Clusters.-Harvard College Observatory Circular, No. 2, announces that an extraordinary number of rariable stars has been discovered in certain globular clusters which have been photographed by Prof. Bailey at Arequipa with the I3-inch Boyden telescope. At least eighty-seven of the stars in the cluster M3 (N.G.C. 5272), in Canes Venatici, have been found to be variable, and in some cases the change of light amounts to two magnitudes or more. In the cluster $M_{5}$ (A.G.C. 5904), forty-six variables were found, out of 750 stars examined, so that they form about six per cent. of the whole of the sixteen stars, contained in a circle $\mathrm{IIO}^{\prime \prime}$ in diameter, six are variable. Smaller numbers of variables have been found in other clusters, but in other cases not a single variable has been detected out of the hundreds of stars which have been photographed; the conditions of the search, however, not taking account of long period changes. In general, no variables have been found within about one minute from the centres of the clusters, on account of the closeness of the stars, and none are more than ten minutes distant from the centres. Some of the newly-discovered variables have short periods, in some cases of only a few hours. Thus, five photographs of X.. (..C. 5904, taken at intervals of an hour on July I, I895, give for the magnitude of a star about three minutes of arc preceding the centre of the cluster, $14.3,13.5,13.8,13.9$, and 14.3 ; four plates, taken at similar intervals on August 9, gave the magnitudes $14^{\circ} 2,14^{\circ} 6,14^{\circ} 8$, and $15^{\circ} \mathrm{O}$.

\section{ON A METHOD OF PHOTOGRAPHY IN NATURAL COLOURS.}

IN I86I Clerk Maxwell described a methor of colour photography, based upon his experiments on the theory of colour vision, and made the following experiment. Three photographs of a coloured object were taken through three several coloured solutions giving images which separately represented the object as it would be seen by each of the three sets of colour nerves postulated by Young. When these were superposed the original colsurs of the object were reproduced, save for the defect that the red and green components suffered from the insensitiveness of the photographic plate of Maxwell's time to the longer wave-lengths. Maxwell added the remark that when the photographic plate was improved as regards sensitiveness to the less refrangible rays, the representation of colour would be improved. ${ }^{2}$

Since Maxwell's day the colour blindness of the plate has been almost completely remedied, thanks to the discovery of Vogel, and it is now possible, proceeding on the lines laid down by Maxwell, to produce by triple projection upon the screen a picture which may be illusively like nature. For the application of modern resources and the suggestion of photographing to the colour vision curves by special colour screens, we have to thank Mr. Ives.

Composite colour photography deals with the subjective reproduction of all visible wave-lengths in two stages; a photographic analysis and an optical synthesis. In the first operation the several wave-lengths are caused to produce three separate photographic images according to their physiological activity in exciting the supposed fundamental red, green, and violet sensations. That is, if the image bears, for example, a yellow colour (suppose such a yellow as the spectral yellow near the D line), one of the plates must record an image of the object having a density of silver deposit corresponding to the degree in which this wave-length can excite the red-seeing nerve, and a second must acquire a density corresponding to the degree in which this same wave-length can excite the green-seeing nerve. The third plate records no impression, for the wave-lengths near $D$ excite no violet sensation; but this yellow sensation is the

1 Abstract of a paper read before the Royal Dublin Society, by Dr. J. Joiy, E.R.S.

On the Theory of Three Primary Colours.' "Collected Papers," p. 449. resultant of two physiological effects only, a red and a green sensation in certain proportions obtained by colour measurements effected upon normal colour sight. We have now obtained three negatives possessing densities of silver deposit corresponding to the degrees in which the three several fundamental colour sensations are stimulated. These degrees of density will be interpreted as degrees of transparency in the positives. The first positive, if backed with a red glass, will transmit a quantity of red light corresponding to the intensity of the physiological excitation of redness in the "red" nerves; the second, backed with green, similarly represents the stimulation of the "green" nerves by the yellow colour of the object; the third positive is backed with blue-violet glass, but is quite opaque, and no violet light is transmitted through it. The projection now of all three images superposed upon the screen forms the second stage of the procedure; the optical synthesis of the original colours. The eye regarding the superposed image receives, in fact, the same amounts of red and green sensation, and experiences the same absence of violet sensation which would have attended the formation of the image of the original object upon the retina.

This process, if accurate reproduction of colour is sought, necessitates the use of two distinct sets of colour selective screens; for the analysing screens will by no means possess the tints ultimately required in the optical synthesis. This is evident since the measurements on colour vision reveal that the wave-lengths near $\mathrm{D}$ are more strongly stimulative of red sensation than are the purely red exciting wave-lengths near $C$, and the wave-lengths again diminish in their power of producing stimulation of the "red" nerves on the more refrangible side of $D$. Hence, in order to photograph the wave-lengths of the spectrum, we require to produce a greater photographic effect by the D wave-lengths than by the $\mathrm{C}$ wave-lengths, and a photographic

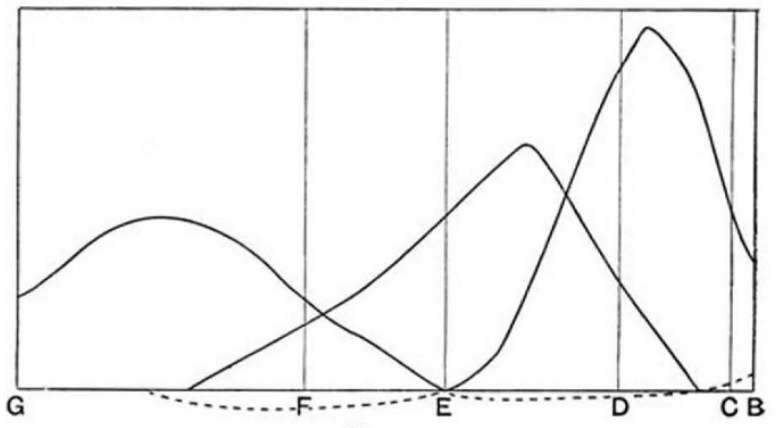

Fig. $x$.

effect diminishing above $\mathrm{D}$ in the same degree as the power of the waves to excite the fundamental red sensation diminishes. To effect this analysis of the light a screen transmitting as predominant wave-length, a wave-length near $D$ must be used for obtaining the image which is to represent the appreciation of light peculiar to the "red" nerves. Such a screen has a yellow-orange colour, which is not the sensation excited in or transmitted by the "red" nerves. In the optical synthesis this must afterwards be represented by a $\mathrm{C}$ red colour. The same remarks apply to the other screens.

Maxwell's curves (Fig. I) are not colour sensation curves (Abney : "Colour Vision," Tyndall Lectures, I895), and it is misleading to speak of the foregoing method as effected on colour sensation curves. Maxwell's curves represent, in fact, the subjective synthesis of the spectrum out of three chosen wave-lengths-a red, a green, and a blue-violet. The question as to how far one or all these chosen wave-lengths may excite more than the one set of nerves remains over, and incleed can only be gone into by examination of abnormal colour vision. In Kœnig's curves of colour vision, colour sensations are plotted. These are shown in the named curves of Fig. 2.

If, from the knowledge afforded by Konig's curves of the compound nature of the green sensation, Maxwell's curves be examined with reference to their suitability to serve the purposes of the photographic method, it will be found that, assuming Maxwell's E green to excite the proportionate amounts of red and violet sensation revealed by Konig's curves, a correct synthesis of the F green by Maxwell's curves is impossible. Although such a comparison is not strictly allowable owing to

NO. I 361 , VOL. 53$]$ 
the red and violet curves of Maxwell being based on different wave-lengths to those used by Kœenig, the fact of grave inaccuracy is certain. This fact will appear if the spectrum is photographed according to Maxwell's curves. The blue-green will then be found to be reproduced too yellow in tone.

In order to apply the colour sensation curves of Kœenig to the photographic method, we have to find by trial examinations of his curves the green most suitable for backing the "green" positive ; for we see that the several green wave-lengths excite very different amounts of red and violet sensation. We find as suitable a wave-length a little to left side of the $\mathrm{E}$ line, about $55^{\circ} \mu \mu$. If we take this colour to back the green positive, we must, in order to find the correct red and violet curves which are to control the densities of the red and violet images, replot the red and violet curves with allowance for the proportionate amounts of red and violet which will be carried to all those points where in the image of the spectrum the green curve operates. The red and violet curves must be lowered by amounts obtained by ascertaining from the height of the green curve at any point the amount of red and violet sensations excited by the amount of our selected green present at that point. The final curves are shown in the slightly altered violet sensation curve, the original green sensation curve, and the considerably lowered red-mixture curve (as it may be called); the full line in all cases representing the applicable curves. It is seen that the amount of negative colour (which cannot be realised) is small. Although it is possible that the compound nature of our green sensations will deny absolute accuracy to this method of colour photography, still my own results on the curves just described, and the results of Ives and others on modified Maxwell curves, appear to show that a degree $o_{f}$

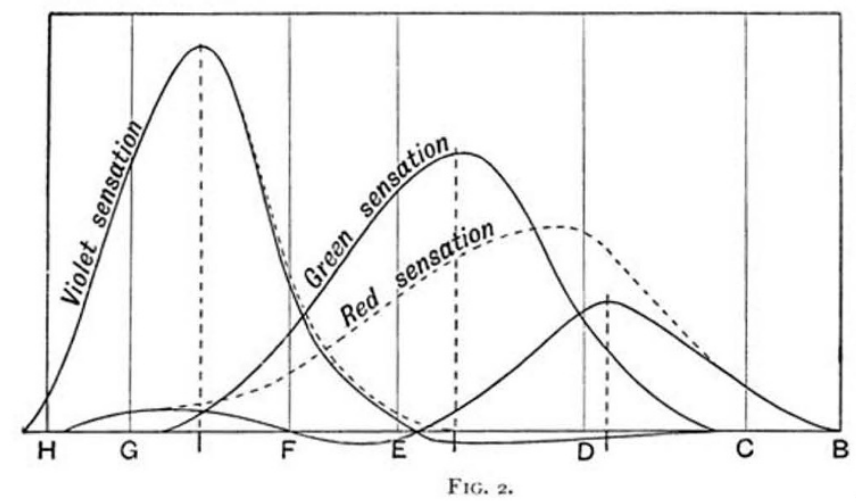

accuracy baffling the criticism of the ordinary untrained eye may be attained, and that in the reproduction of the most complex tints.

The symmetry of the derived curves renders their application easy. The transmission of light through a pigment is not limited generally to a small group of predominant wave-lengths, but falls off uniformly at either side in the directions of longer and shorter waves. If we choose the pigments used on the analysing screens so that their predominant transmissions are at three points in the spectrum indicated by the axis of symmetry of the three curves, these being nearly symmetrical, very accurate results are obtained. The positions of these axes of symmetry are shown by the vertical dotted lines. Accordingly, I make the colour of the red-taking screen that of the spectrum at a point displaced to the red side of $\mathrm{D}$ by about one-sixth the interval $\mathrm{D}$ to $\mathrm{C}$; for the green and violet-taking screens the correct tints are found in the same manner by scaling from the figure. Good results are thus obtained, but I do not assert that these details of procedure are final.

Any method of photography in natural colours must possess the characteristics not only of accuracy of colour rendering, but also of convenience of application and permanency of colour, if it is to possess value as a scientific method. For use under the various circumstances of travel the naturalist requires a method no more cumbersome than the present dry plate. In the method of composite colour photography, as described, the ordinary camera will not serve. The cumbersome necessity of obtaining three images remains, and subsequently no concrete

$$
\text { NO. I } 36 \text { I, VOL. } 53 \text { ] }
$$

image in natural colours is actually obtained. One can only be realised by triple projection upon a screen, or by using some optical contrivance which, by the aid of reflectors, enables all three images to be simultaneously projected upon the retina.

I now proceed to describe a mode of applying the foregoing principles which is free of the objection of cumbersomeness, and which enables us to realise a concrete image in transparent colours. A plate is finally produced which may be held in the hand, regarded against the light, and which bears an image of the object in natural colours, or such as are so nearly accurate as to seem so to the eye. In this new method there is but the one image photographed. The ordinary camera, lens, and backs, \&c., are used without modification. The first-class isochromatic plates in the market, which are sensitised down to the $\mathrm{C}$ red, will give very good results.

In the new method the idea is to carry the application of physiological principles still further, and divide up the plate like a hypothetical subdivision of the retina, so that all over the plate there should be minute regions uniformly distributed wherein the sensitive silver salt is excited to become reduced to the " photogenic" material in the same degree in which the sensations of redness, greenness, violetness, would have been actually excited in the several nerves of the retina had the image been formed upon it. Development builds upon this photogenic material the denser silver deposit, and ultimately in the positive the amounts of the sensations are registered in the degrees of transparency of the successive regions. The lined screen which can bring about this I can show you in the microscope. It consists of closely ruled adjacent lines in orange, green and violet tints. This screen, applied closely to the sensitive surface, analyses the image in the camera. The screens I have used hitherto are coarse, about 200 lines to the inch, and even with this coarseness will show plainly, I regret to say, the imperfections of the only apparatus at my com. mand in preparing these screens. I may observe, in passing, that the colours are ruled on in pigments made up as inks in gelatine and gum arabic or dextrine, and upon plates coated with a preliminary layer of gelatine. Such lines may be put on so close as 800 or 1000 to the inch. With between 300 and 400 to the inch, however, the eye is no longer annoyed by the structure of the plates. The lines may also be ruled on celluloid or on translucent paper.

The appearance of both negative and positive obtained is interesting. One would hardly at first sight distinguish between them and the ordinary images. But a lens readily shows the difference. Recalling now that the lines upon the positive register in their degrees of transparency the degrees in which the three-colour sensations would have been excited, it becomes apparent that to complete the physiological parallel we must convert these degrees of transparency to quantities of the red, blue and violet colour sensations. This is done by a second screen, which carries red, green and violet lines to the same gauge as the taking screen. We apply this to the positive, and as we move it over the image, waves of every tint of colour appear till that position is reached where the red lines fall over the lined areas recording red sensation, and so for the others. The picture now suddenly appears in vivid colour and with all the realism and relief conferred by colour perspective.

A picture of wallflowers taken through a dichromatic screen, the red and green sensations only being photographed, is of interest as realising the appearance of the object to a violet-blind eye. The rich reds and browns appear unaffected; the greens are, however, somewhat unnatural. A photograph of the spectrum shows the range of colour from the $\mathrm{C}$ red to the $\mathrm{H}$ lines. The blue-green is, however, defective. It was taken according to Maxwell's curves. Photographs of burnished metallic objects, as a brightly lacquered microscope, reproduce the metallic lustre ; and one of an uranium glass bowl, reproduces the characteristic dichroism and fluorescent appearance of the glass when seen by daylight. That every shade of colour can be reproduced, how. ever complex, is shown by two portraits, one from life, and one a copy of a water-colour drawing boasting very æsthetic shades of brown and olive. A great variety of bright sunlit colours appear in a view at the Trinity College athletic sports, wherein the scarlet uniforms of the military band, the green of the grass, and the blue sky, recall the vivid appearance of the image on the ground glass screen of the camera. The colour perspective in such pictures adds greatly to the reality and relief. The faithful reproduction of texture, as in the case of some pansies, where the velvety browns and purples of the originals reappear, or as in the case of the wallflowers, reminds us how much is 
inferred from the most subtle differences of light and shade in the colours of objects, in association with previous experience derived through other senses. The picture is always an optical illusion ; and this additional illusiveness conferred on the photograph by the method invented by Maxwell on the basis of the inree-colour theory of vision, is surely a strong confirmation of that theory.

These results are attained by no new photographic operations. It is necessary to use good orthochromatic plates sensitised into the red, and also to have affixed in the lens an orthochromatic screen cutting of the ultra-violet light in the usual manner. The exposure is somewhat longer than the ordinary exposure, for we can of course only use visible light, and of this a part is stopped by the taking screen. The ordinary backs may be used. The displacement of the sensitive film from accurate register with the ground glass camera screen, owing to the presence of the taking screen in front of it, may be corrected (if thought necessary) by simply reversing the surface of the ground glass camera screen, turning the muffled side outward. This secures that the image will be accurately focussed in the plane of the sensitive surface. Negatives and positives may be used as ordinary negatives or positives till it is desired to recall the original colours. Thus, for those who wander with the camera, the possession of but the one seeing screen to test results is sufficient, and of course the one taking screen suffices to take an indefinite number of plates.

These considerations lead us naturally to observe that the registration of colour being really carried in the silver image, which with very little care in manipulation may be made permanent, secures that the colours are permanent. A faded screen may at any time be made good by a fresh screen ; the colours in all cases being spectroscopically chosen, we are assured of the reproduction of the original colour. In this aspect the necessity of the detached colour screen is no disadvantage, but rather a necessary safeguard against the inevitable fading attending most pigment colours.

\section{COMET MAGNITUDES.}

$D^{\mathrm{R}}$. HOLETSCHEK, of the Vienna Observatory, has recently communicated to the Imperial Academy of Sciences a paper on the magnitude and brilliancy of comets and their tails, with the view of arranging them in "magnitudes" or orders of brilliancy in a manner similar to that in which stars are arranged according to their lucidity. Further, from the data given as to the apparent length of the tail, the true length of the tail has been computed, and an inquiry instituted as to the possibility of tail formation and its probable length, based on the resulting magnitude of the comet and its perihelion distance.

If it be true that the brilliancy of a comet varies as the squares of the distances from the sun $(r)$, and from the earth $(\Delta)$, then from observations made at various points of the orbit, the same "magnitude" ought to result for the values $r=\Delta=\mathrm{I}$. This magnitude Dr. Holetschek has deduced, where sufficient data existed, and the results can be practically arranged in two classes : one, in which the deduced magnitudes derived from various values of $r$ and $\Delta$ so nearly agree that a mean can be taken; the other, in which is shown a regular progress, and always in the direction that the deduced magnitudes with small radii vectures, therefore when the comet is near perihelion, are greater than when at large distances from the sun. The origin of this is due to the fact that the second power of the radius vector does not fully represent the variations occurring in comets as they approach the sun, at which time their brilliancy is more increased than is shown by the ratio I : $r^{2} \Delta^{2}$. The first case, it is suggested, is only a special case of the second, arising through insufficiency of description. This is most clearly shown when the time covered by the observations is short, or the details so wanting in accuracy that the deviation from the ratio $\mathrm{I}: r^{2} \Delta^{2}$ cannot be known with certainty. The conclusion drawn is that the formula so generally used can represent the brilliancy of a comet at different distances from the sun for a short time only, and is inapplicable for long periods.

Dr. Holetschek uses the deduced magnitude in the neighbour hood of the perihelion, valuable as showing the greatest brilliancy attainable in a particular comet, as the data for forming the comets into orders of magnitude, and inquires how far the tail formation is connected with this magnitude and the perihelion distance. He decides from his material, that when the deduced NO. I $36 \mathrm{I}$, VOL. 53$]$ magnitude is 6 or lower than 6 , only a short and feeble tail, or one not visible to the naked eye, is possible. Comets with a deduced magnitude of 4 , or still brighter, have a tail well visible, which is the greater, the smaller the perihelion distance, and the smaller, the greater this distance. Within the limits between 4 and 5 magnitude, if we exclude very great perihelion distances, lie the possibilities of a considerable tail development.

Dr. Holetschek has also considered the diameters of comets, and sought to introduce order by reducing the apparent diameter to that corresponding to a distance of the comet from the earth equal to unity. In the case of Halley's comet, no diminution or variability is to be detected in either its brilliancy or the length of its tail. The same values serve from 1456 to 1835 . As, however, on account of the continual development of the tail, diminution of the mass is probable, it cannot be decided whether the approximate constancy, shown in the investigation, arises from inadequacy in the observations themselves, or is produced by certain processes existing in cometary bodies.

\section{UNIVERSITY AND EDUCATIONAL INTELLIGENCE.}

OxForD. - The delegates of the Common University Fund have elected Mr. Edwin Stephen Goodrich, Merton College, to the Biological Scholarship at Naples for the year I 895-96.

CAMBRIDGE.-The report to the Senate of the Engineering Laboratory Syndicate, dated November 9, I895, is a very satisfactory record of energetic work. The University was unable to provide more than $£$ Iooo for the building and equipment of the laboratory, which was estimated to cost about $£ 6000$. By steady and enthusiastic efforts Prof. Ewing and his colleagues succeeded in collecting from many sources, within and without the University, the $£ 5000$ that were needed; and what is still more to their credit, have completed the work in hand with a small balance to the good. The continued growth of the department, in which there are now eighty-five students under instruction, makes a further extension of the buildings urgently necessary. A site has been provided for the purpose, but further funds are needed for construction. The workshops, never intended to be other than temporary, must soon be rebuilt, and more lecture-room accommodation must be provided. The department has certainly justified its existence, and the Syndicate have proved themselves to be worthy stewards of the funds placed at their disposal. It is to be hoped that, with these guarantees that they will be well used, the needful moneys may ere long be forthcoming. The valuable services of Mr. Dalby and Mr. Lamb, the demonstrators of mechanism and engineering, in the work of organising the laboratory, are mentioned with cordial appreciation in the report. Among the donors of contributions of over $£$ ioo are the Duke of Devonshire, the late Earl of Derby, Mr. Frank McClean, and Dr. John Hopkinson, and eleven benefactors have given $f_{\mathrm{I}}$ oo each. Valuable donations of apparatus and of books have helped greatly towards the furnishing of the laboratory.

The amount, clear of all expenses, available for the Robertson Smith memorial, is $f_{1} 45^{\circ}$. It has been agreed by the subscribers that sufficient of this amount should be invested to produce an annual income of $£ 30$, such income to be employed on the continuance and extension of Prof. Robertson Smith's library, which he bequeathed to Christ's College. It was also resolved that the remainder of the amount collected, after all expenses have been paid, be handed over to the University for the purchase of Oriental MSS. for the University Library, which shall be marked as having been acquired by means of the fund. It is estimated that about $£ 3^{00}$ will be handed over to the Syndics of the Library.

IT is reported that Mr. P. N. Russell has given the sum of $£ 50,000$ to endow a school of engineering in connection with the Sydney University.

AN address on the present state and position of technical instruction in this country, delivered by Major-General Sir John Donnelly before the Society of Arts on Wednesday, November 20 , is printed in full in the current Fournal of the Society.

THE annual meeting of the National Association for the Promotion of Technical and Secondary Education, and the Conference of Representatives of Technical Education Committees, will be held on Tuesday, December Io, at the Royal United Service Institution, Whitehall. 